\title{
Environment humidity and temperature prediction in agriculture using Mamdani inference systems
}

\author{
Julio Barón Velandia, Jonathan Steven Capera Quintana, Sebastian Camilo Vanegas Ayala \\ Facultad de Ingeniería, Universidad Distrital "Francisco José de Cáldas," Colombia
}

\begin{tabular}{l} 
Article Info \\
\hline Article history: \\
Received Jul 20, 2020 \\
Revised Oct 21, 2020 \\
Accepted Dec 5, 2020
\end{tabular}

\section{Keywords:}

Fuzzy set

Greenhouse

Humidity prediction

Optimization

Temperature prediction

\begin{abstract}
This paper presents the results of a humidity and temperature prediction model in the environment for agriculture, using diffuse sets and optimizing their parameters by heuristic methods, such as genetic algorithms, and exact methods such as Quasi-Newton. It has been identified that non-specialized users could have difficulties in understanding the system dynamics and the behavior of variables over time. The aim of this research is obtain models with a high level of interpretability and accuracy that allows predicting the temperature and humidity values for the environment. The use of fuzzy logic to present a solution has great advantages as this system is highly rated for interpretability. Furthermore, by relating the obtained values for environment humidity and temperature to qualitative categories as high, medium or low, it allows non-specialized users to have a better understanding of the system dynamics. Two optimization techniques are applied to two different diffuse sets that allow the prediction of the humidity and temperature. It is found that the best implementation involves a Mamdani fuzzy inference system optimized with Quasi-Newton algorithm that uses a set of initial values attained through a previous optimization process with a genetic algorithm.
\end{abstract}

This is an open access article under the $\underline{C C B Y-S A}$ license.

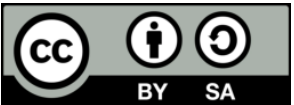

\section{Corresponding Author:}

Sebastian Camilo Vanegas Ayala

Facultad de Ingeniería

Universidad Distrital "Francisco José de Cáldas"

Carrera 7 No. 40B - 53, Bogotá D.C., Colombia

Email: scvanegasa@correo.udistrital.edu.co

\section{INTRODUCTION}

As humankind's part in agriculture evolves from survival and circumstantial crops to commercial ones, the system complexity evolves too, and the degree of knowledge required for successful cropping increases [1]. According to the changes on the climate, the best-suited areas for any crop can be modified and multiple variables like the temperature and humidity of the zone that can affect the crops' performance, and its impact on the soil use, the biodiversity, the region's socio-economics, the agricultural production, and other, must be carefully evaluated [2]. Bearing in mind the massive application of the information and communication technologies (ICT), its influence on agricultural production, and how it gathers the products of the internet and big data eras [3], management and environmental information during the cropping process play a key role in the prediction of fore-coming climate changes [4].

A web applied monitoring system that uses sensors located in strategic places along a plant field allows users to monitor the crop status anywhere, and anytime, from any device with remote access capabilities [5]; therefore, the prediction on variables as temperature and humidity can be made from different data attained from similar systems. Nevertheless, such forecasting requires efficient algorithms as the Stochastic gradient descent algorithm that would give it a greater accuracy, hence, it is necessary to apply 
different optimization techniques, heuristic or exact, to improve alike models [6]. Ensuring a certain degree of interpretability is as important as accuracy so it could be understood by any person if needed, to accomplish that aim the use of an intuitive Mamdani fuzzy inference systems (configured by multiple conditional rule sets) could be a suitable solution [7,8].

Neural networking, usually used in black box systems, is a technology that also contributes to the study of the dynamic behavior of climate; in the agricultural field, its application eases the water use balance in irrigation $[9,10]$. Furthermore, computer models capable of predicting climate behavior and the impact of greenhouse gases on earth's temperature were developed some decades ago, however, these models only analyze the $\mathrm{CO} 2$ related variables [11], presence, and concentration, when research has shown there are many others involved [12]. The most recent models to predict temperature and humidity related variables are mathbased (using Navier-Stokes equations) or computer-based (simulations on the earth's physics for the atmosphere and oceans), both, commonly used to study climate change based on global predictions over atmospheric and oceanic phenomena [13-17]. As developed as the field appears to be, there are only a few models for both temperature and humidity prediction focused on local settings, some of these use just one model type (usually through mechanisms or experience) targeted for small mediums with equipment for heating and humidity control [18].

This research intends to combine a fuzzy inference system for both temperature and humidity prediction with an optimization technique to improve the interpretability of the fore-mentioned techniques and generate adequate data for the management of these variables. The use of Mamdani fuzzy inference systems allows a high degree of interpretability that is necessary for better understanding by non-specialized users $[7,8]$. The remainder of the paper is organized as section 2 research method, section 3 results and discussions and section 4 conclusion.

\section{RESEARCH METHOD}

This research is carried out in two stages: the first stage comprehends a process of data recollection while the second stage involves a proposal development process within an iterative and increasing short life cycle as shown in Figure 1. The proposal development follows a prototyping paradigm due to the uncertainty of the performance of the algorithms used [19].

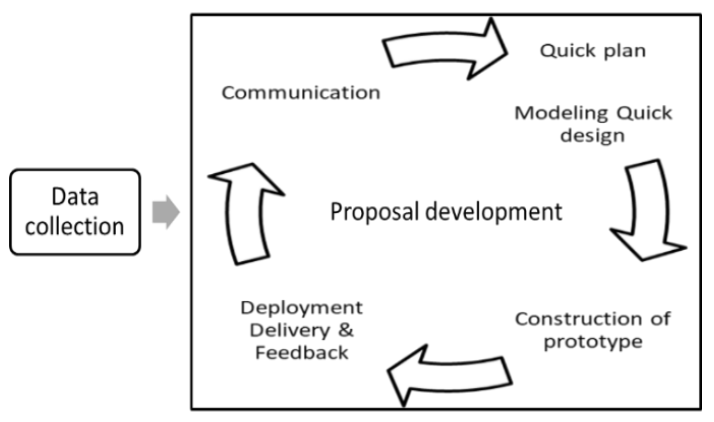

Figure 1. The employed research method (adapted from [19])

\subsection{Data recollection}

For this research, the input set is determined by the measure values for both temperature and humidity registered 48 hours before the prediction day, and for the output set, the values were obtained during the prediction day. All the values were taken from the periodic register for Bogotá, Colombia, made by "Instituto de Hidrología, Meteorología y Estudios Ambientales" (IDEAM) [20].

\subsection{Proposed methods}

Two proposals based on Mamdani fuzzy inference systems are used for this research, both will be optimized whether through a genetic algorithm or a Quasi-Newton algorithm [21-23]. The proposal development process follows a quick method for evolutive models based on software prototyping [19] comprised for five steps: Communication, quick planning, modeling and quick design, prototype construction and application, delivery and feedback.

- Communication: The requirements set by the stakeholders are concealed.

- Quick planning: The route book to follow is defined according to the proposed schedule. 
- Modeling and quick design: The characteristics and parameters for the fuzzy inference systems are set to foster an adequate implementation and a correct optimization.

- Prototype construction and application: If the design is feasible, the proposal is tested using the dataset and the predicted values for both temperature and humidity are verified using a MATLAB toolbox [24]. If any requirement cannot be fulfilled or it is necessary to add any function to the system, another complete cycle for the previous stages will be required.

At the end of the development process, mean squared error (MSE) defined as $M S E=\frac{1}{n} \sum_{j=1}^{n}\left(y_{j}-\right.$ $\left.\widehat{y_{J}}\right)$ and the effectiveness rate for each proposal is calculated in every variable as Effectiveness $(\%)=$ $(1-\sqrt{M S E}) * 100$. This final step would determine the best prediction model in this work [25].

\section{RESULTS AND DISCUSSION}

The Mamdani fuzzy inference system uses a centroid defuzzification. The model is made up of three fuzzy sets with three membership functions and a set of rules that relates them all and describes the system. The initial parameters of the fuzzy sets in the genetic algorithm were defined randomly from a range between minimum and maximum values determined by statistical analysis. The parameters in the Quasi-Newton algorithm were obtained by the best performance of the genetic algorithm. This excerpt presents the results for both model proposals and the comparison of their outcomes on the prediction of the values for both temperature and humidity.

\subsection{Mamdani fuzzy inference system optimized with genetic algorithm}

The genetic algorithm is set to a 50 individuals population, 200 generations, and an initial population range between $|0.6,2|$ for temperature measured in Celsius degrees and $|0.5,1|$ for percent relative humidity. The fitness function, for both variables, is defined as the MSE for normalized data. The Mamdani system in which the algorithm is applied possesses two inputs divided into triangular type sets (trimf) and a triangular output for both variables. The optimization method was applied to a set of 27 parameters that represent the values of the points of the triangular membership functions. After 30 iterations, the minimum and maximum errors, as well as the MSE, are calculated for each iteration. The average MSE for humidity and temperature are $9.09 \mathrm{E}-04$ and $5.39 \mathrm{E}-03$. with standard deviations of $2.80 \mathrm{E}-40$ and $1.95 \mathrm{E}-03$, respectively. The series, E1..., E30, correspond to the genetic algorithm iterations with random configurations in every execution and $\bar{x}$ relates to the mean value of each column as shown in Table 1.

The comparison between the predicted and obtained humidity data is shown in Figure 2, where the $\mathrm{x}$-axis represents the period analyzed ( 24 hours) and the y-axis the humidity values. As observed in the Figure 2, iteration E19 had the best performance values as presented in Table 1. E19 also reports an MSE of 4.24 E-04 as shown in Figure 3, where the error through time relationship for the predicted and obtained humidity data oscillates between 0.06 and 0.03 . The comparison between the predicted and obtained temperature data is shown in Figure 4, where the iteration E26 shows the best performance values see in Table 1 and an MSE of 2.84 E-03. Figure 5 shows the error through time relationship for the predicted and obtained temperature data oscillates between 0.1 and -0.2 .

\subsection{Mamdani fuzzy inference system optimized with Quasi-Newton algorithm}

The Quasi-Newton algorithm is set to an initial configuration based on the feedback from the aforementioned Mamdani fuzzy inference system optimized with a genetic algorithm. The optimization method was applied to a set of 27 parameters that represent the values of the points of the triangular membership functions. The fitness function for both variables is defined as the MSE for normalized data. The minimum, maximum, and average error values for this configuration are shown in Table 2.

The comparison between the predicted and obtained humidity data for the period analyzed (24 hours) is shown in Figure 6. Figure 7 presents the model MSE, 1.21 E-04, and the error through time relationship for the predicted and obtained humidity data that oscillates between 0.02 and -0.03 . The comparison between the predicted and obtained temperature data for the period analyzed ( 24 hours) is shown in Figure 8. Figure 9 presents the model MSE, $2.03 \mathrm{E}-03$, and the error through time relationship for the predicted and obtained humidity data that oscillates between 0.01 and -0.15 .

\subsection{Results' comparison}

Table 3 shows the results and effectiveness percentages (calculated from the RMSE) for both configurations; the best performance configuration is the proposal for Mamdani fuzzy inference system optimized with a Quasi-Newton algorithm: 1.21 E-04 MSE for humidity values and 2.03 E-03 MSE for temperature values. 
Table 1. Genetic algorithm iterations in a Mamdani fuzzy inference system (C1)

\begin{tabular}{|c|c|c|c|c|c|c|}
\hline & \multicolumn{3}{|c|}{ HUMIDITY } & \multicolumn{3}{|c|}{ TEMPERATURE } \\
\hline & MIN & MAX & MSE & MIN & MAX & MSE \\
\hline E1 & $1.41 \mathrm{E}-03$ & $5.79 \mathrm{E}-02$ & $1.08 \mathrm{E}-03$ & $3.39 \mathrm{E}-03$ & $1.36 \mathrm{E}-01$ & $4.97 \mathrm{E}-03$ \\
\hline E2 & $1.38 \mathrm{E}-03$ & $6.46 \mathrm{E}-02$ & 7.35 E-04 & $1.65 \mathrm{E}-03$ & $2.01 \mathrm{E}-01$ & $6.02 \mathrm{E}-03$ \\
\hline E3 & $2.53 \mathrm{E}-05$ & $1.01 \mathrm{E}-01$ & $1.19 \mathrm{E}-03$ & $0.00 \mathrm{E}+00$ & $1.74 \mathrm{E}-01$ & $5.60 \mathrm{E}-03$ \\
\hline E4 & $4.42 \mathrm{E}-03$ & $6.08 \mathrm{E}-02$ & $1.49 \mathrm{E}-03$ & $1.45 \mathrm{E}-03$ & $1.62 \mathrm{E}-01$ & 7.85 E-03 \\
\hline E5 & $3.25 \mathrm{E}-03$ & 6.17 E-02 & 1.15 E-03 & $1.32 \mathrm{E}-02$ & 1.54 E-01 & 9.68 E-03 \\
\hline E6 & $5.00 \mathrm{E}-03$ & $5.79 \mathrm{E}-02$ & 9.92 E-04 & $1.83 \mathrm{E}-02$ & $1.93 \mathrm{E}-01$ & $5.65 \mathrm{E}-03$ \\
\hline E7 & $2.58 \mathrm{E}-04$ & $4.55 \mathrm{E}-02$ & $6.19 \mathrm{E}-04$ & $2.01 \mathrm{E}-03$ & $1.40 \mathrm{E}-01$ & $5.65 \mathrm{E}-03$ \\
\hline E8 & $3.20 \mathrm{E}-03$ & $4.90 \mathrm{E}-02$ & $9.81 \mathrm{E}-04$ & $0.00 \mathrm{E}+00$ & $1.54 \mathrm{E}-01$ & $5.28 \mathrm{E}-03$ \\
\hline E9 & $5.24 \mathrm{E}-03$ & $7.00 \mathrm{E}-02$ & $1.45 \mathrm{E}-03$ & $5.42 \mathrm{E}-04$ & 2.22 E-01 & $1.10 \mathrm{E}-02$ \\
\hline E10 & $1.29 \mathrm{E}-03$ & $1.00 \mathrm{E}-01$ & $1.38 \mathrm{E}-03$ & $0.00 \mathrm{E}+00$ & $2.25 \mathrm{E}-01$ & $5.91 \mathrm{E}-03$ \\
\hline E11 & $1.78 \mathrm{E}-03$ & $5.93 \mathrm{E}-02$ & 7.17 E-04 & $6.59 \mathrm{E}-04$ & $1.36 \mathrm{E}-01$ & $3.98 \mathrm{E}-03$ \\
\hline E12 & $1.64 \mathrm{E}-03$ & $5.31 \mathrm{E}-02$ & 6.03 E-04 & $5.13 \mathrm{E}-03$ & $1.20 \mathrm{E}-01$ & $3.12 \mathrm{E}-03$ \\
\hline E13 & $2.93 \mathrm{E}-03$ & $4.35 \mathrm{E}-02$ & 4.97 E-04 & $1.22 \mathrm{E}-03$ & $1.68 \mathrm{E}-01$ & $5.85 \mathrm{E}-03$ \\
\hline E14 & $6.62 \mathrm{E}-04$ & $9.71 \mathrm{E}-02$ & 1.04 E-03 & $7.38 \mathrm{E}-04$ & $1.61 \mathrm{E}-01$ & $4.00 \mathrm{E}-03$ \\
\hline E15 & 6.54 E-05 & 8.00 E-02 & $9.56 \mathrm{E}-04$ & 3.96 E-04 & $1.50 \mathrm{E}-01$ & 3.02 E-03 \\
\hline E16 & $2.48 \mathrm{E}-04$ & 7.86 E-02 & $1.26 \mathrm{E}-03$ & 2.44 E-03 & 1.17 E-01 & 3.69 E-03 \\
\hline E17 & $1.40 \mathrm{E}-03$ & 8.01 E-02 & 9.86 E-04 & 9.35 E-04 & $1.20 \mathrm{E}-01$ & $3.78 \mathrm{E}-03$ \\
\hline E18 & 4.32 E-04 & 6.45 E-02 & $1.08 \mathrm{E}-03$ & $1.11 \mathrm{E}-02$ & $1.35 \mathrm{E}-01$ & $5.90 \mathrm{E}-03$ \\
\hline E19 & 9.06 E-04 & 5.03 E-02 & 4.24 E-04 & 1.02 E-03 & 1.32 E-01 & 4.72 E-03 \\
\hline E20 & 8.27 E-04 & $6.11 \mathrm{E}-02$ & 8.80 E-04 & $0.00 \mathrm{E}+00$ & $1.65 \mathrm{E}-01$ & $6.10 \mathrm{E}-03$ \\
\hline E21 & 7.37 E-04 & $9.59 \mathrm{E}-02$ & $1.02 \mathrm{E}-03$ & $4.62 \mathrm{E}-03$ & $1.40 \mathrm{E}-01$ & $4.83 \mathrm{E}-03$ \\
\hline E22 & $1.30 \mathrm{E}-03$ & $4.70 \mathrm{E}-02$ & 5.92 E-04 & $5.65 \mathrm{E}-04$ & 1.73 E-01 & $4.70 \mathrm{E}-03$ \\
\hline E23 & $6.09 \mathrm{E}-03$ & 8.29 E-02 & 1.02 E-03 & 7.26 E-03 & 1.82 E-01 & 8.05 E-03 \\
\hline E24 & 1.94 E-04 & 7.38 E-02 & $6.69 \mathrm{E}-04$ & 7.44 E-03 & 1.97 E-01 & $4.19 \mathrm{E}-03$ \\
\hline E25 & $6.89 \mathrm{E}-03$ & $6.91 \mathrm{E}-02$ & $6.30 \mathrm{E}-04$ & $0.00 \mathrm{E}+00$ & 1.80 E-01 & 3.65 E-03 \\
\hline E26 & 1.90 E-03 & 9.42 E-02 & 8.92 E-04 & 2.96 E-03 & $1.56 \mathrm{E}-01$ & 2.84 E-03 \\
\hline E27 & $1.05 \mathrm{E}-03$ & 7.25 E-02 & $6.24 \mathrm{E}-04$ & $5.70 \mathrm{E}-03$ & $1.49 \mathrm{E}-01$ & 7.73 E-03 \\
\hline E28 & $1.06 \mathrm{E}-03$ & $6.26 \mathrm{E}-02$ & $6.91 \mathrm{E}-04$ & 8.17 E-03 & 1.13 E-01 & 4.44 E-03 \\
\hline E29 & 7.43 E-03 & $5.20 \mathrm{E}-02$ & 7.70 E-04 & 4.01 E-03 & 1.19 E-01 & 3.13 E-03 \\
\hline E30 & $1.83 \mathrm{E}-04$ & 7.64 E-02 & 8.64 E-04 & 1.42 E-03 & $1.29 \mathrm{E}-01$ & 6.40 E-03 \\
\hline \multirow[t]{2}{*}{$\overline{\mathbf{x}}$} & 2.11 E-03 & 6.87 E-02 & 9.09 E-04 & 3.54 E-03 & 1.57 E-01 & 5.39 E-03 \\
\hline & \multicolumn{2}{|c|}{ STANDARD DEVIATION } & 2.80 E-04 & \multicolumn{2}{|c|}{ STANDARD DEVIATION } & 1.95 E-03 \\
\hline
\end{tabular}

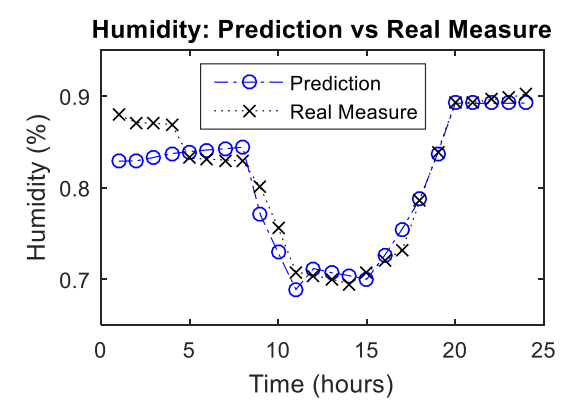

Figure 2. Humidity data comparison (Mamdani GA)

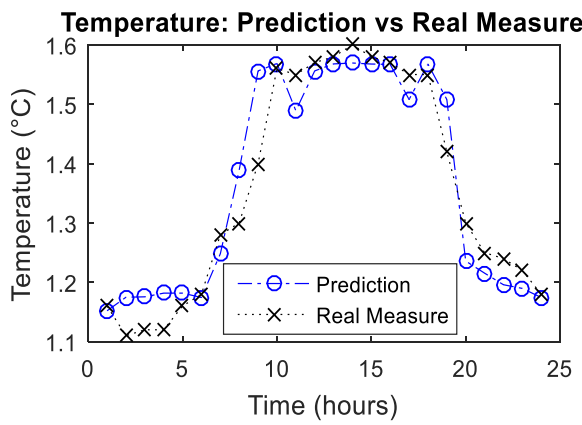

Figure 4. Temperature data comparison (Mamdani GA)

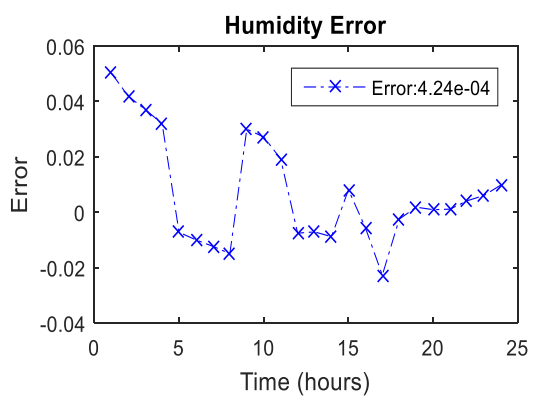

Figure 3. Error-values for humidity data (Mamdani GA)

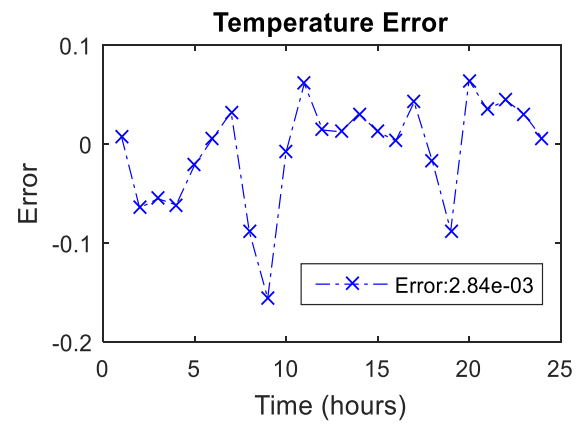

Figure 5. Error-values for temperature data (Mamdani GA) 
Table 2. Quasi-Newton optimization on the Mamdani fuzzy inference system

\begin{tabular}{ccc}
\hline Error & Humidity & Temperature \\
\hline MIN & $4.58 \mathrm{E}-04$ & $1.07 \mathrm{E}-03$ \\
MAX & $2.20 \mathrm{E}-02$ & $1.13 \mathrm{E}-01$ \\
MSE & $1.21 \mathrm{E}-04$ & $2.03 \mathrm{E}-03$ \\
\hline
\end{tabular}

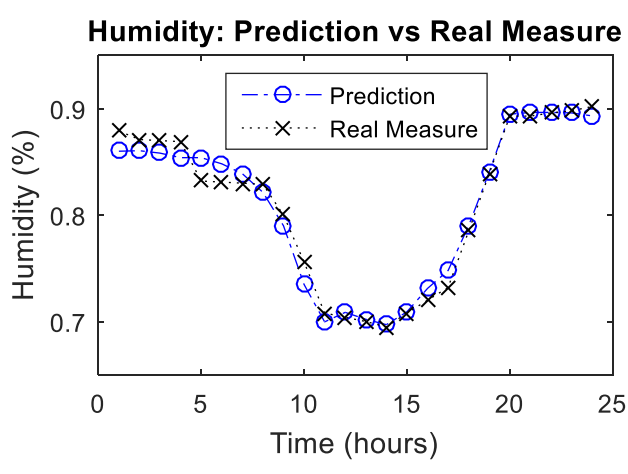

Figure 6. Humidity data comparison (Mamdani QN)

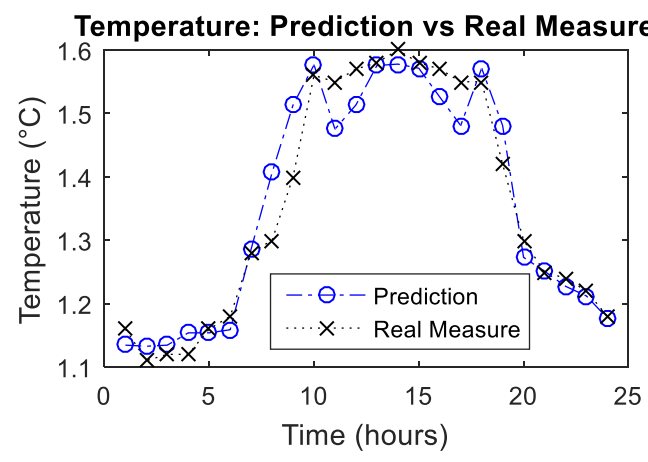

Figure 8. Temperature data comparison (Mamdani QN)

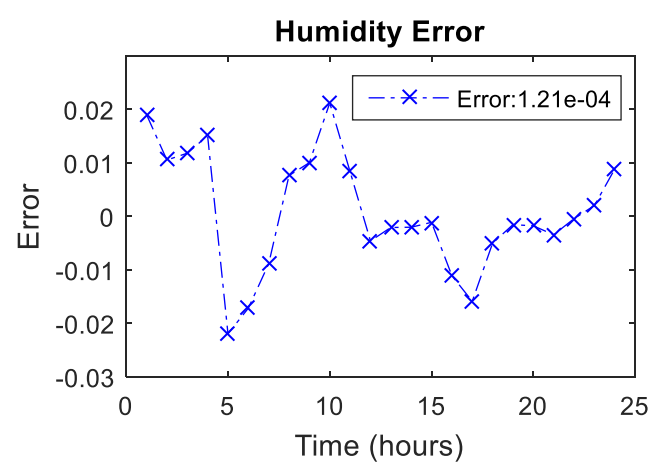

Figure 7. Error-values for humidity data (Mamdani QN)

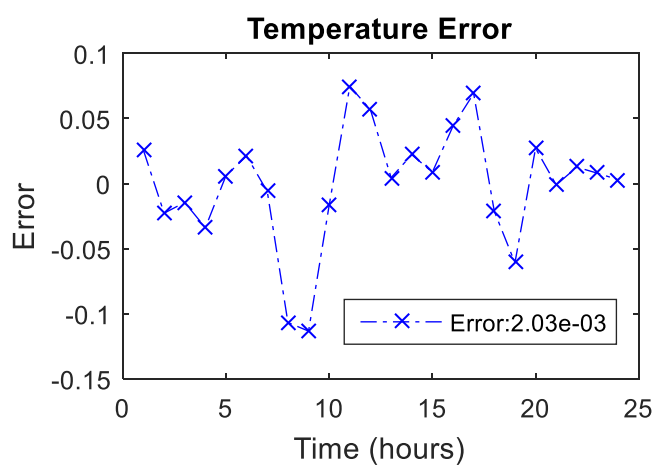

Figure 9. Error-values for temperature data (Mamdani QN)

Table 3. Environment humidity and temperature analysis

\begin{tabular}{lcccc}
\hline \multicolumn{1}{c}{ PROPOSAL } & HUMIDITY MSE & $\begin{array}{c}\text { TEMPERATURE } \\
\text { MSE }\end{array}$ & $\begin{array}{c}\text { HUMIDITY } \\
\text { EFFECTIVENESS (\%) }\end{array}$ & $\begin{array}{c}\text { TEMPERATURE } \\
\text { EFFECTIVENESS (\%) }\end{array}$ \\
\hline $\begin{array}{l}\text { Mamdani fuzzy inference } \\
\text { system optimized with } \\
\text { genetic algorithm }\end{array}$ & $4.24 \mathrm{E}-04$ & $2.84 \mathrm{E}-03$ & 97.94 & 94.67 \\
$\begin{array}{l}\text { Mamdani fuzzy inference } \\
\text { system optimized with } \\
\text { Quasi-Newton algorithm }\end{array}$ & $1.21 \mathrm{E}-04$ & $2.03 \mathrm{E}-03$ & 98.9 & 95.49 \\
\hline
\end{tabular}

Figure 10 shows the humidity Mamdani fuzzy inference system optimized with a Quasi-Newton algorithm, where (a) is the first-day input sets; (b) is the second-day input sets; (c) is the output set that represents the third-day prediction. The mentioned sets are related through qualitative ranges for humidity (high, medium, low) and their membership degree between zero and one for each domain. Figure 11 shows the temperature Mamdani fuzzy inference system optimized with a Qasi-Newton algorithm, where (a) is the first-day input sets; (b) is the second-day input sets; (c) is the output set that represents the third-day prediction. The mentioned sets (defined using membership functions type trimf) are related through qualitative ranges for temperature (high, medium, low).

The correspondence between humidity and temperature values obtained from the set of rules, as well as its interaction with the fuzzy sets generated by the algorithm, is graphically described in the 
Figures 12 and 13. Figures 14 and 15 present surface figures that exemplify how the fuzzy set definition for humidity and temperature values, and the rule set for prediction on the third-day work based on the measures taken the two previous days.

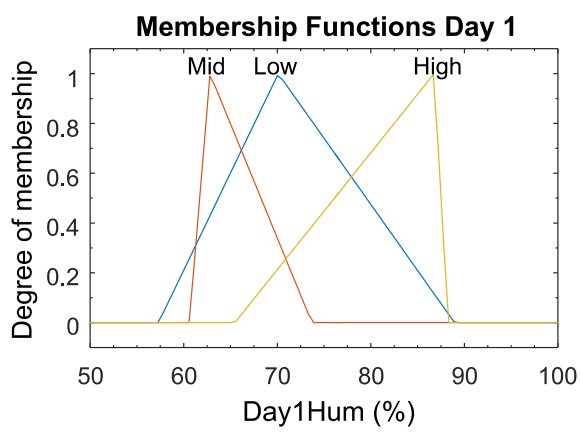

(a)

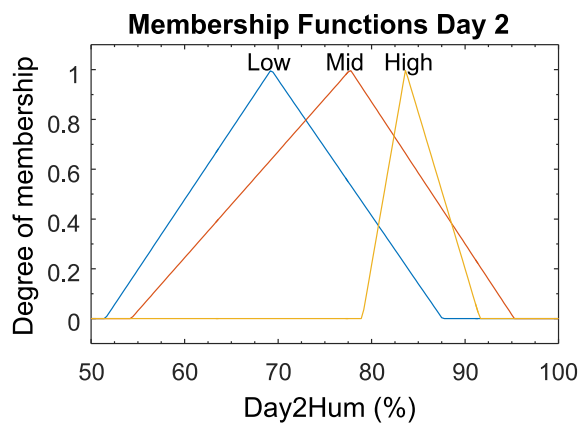

(b)

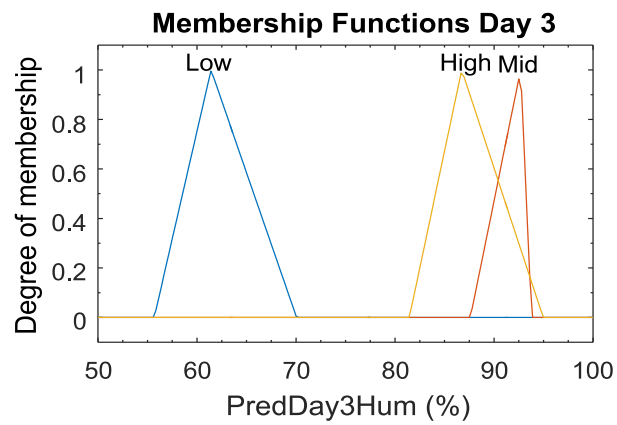

(c)

Figure 10. Membership functions for environment humidity: (a) day 1; (b) day 2; (c) day 3

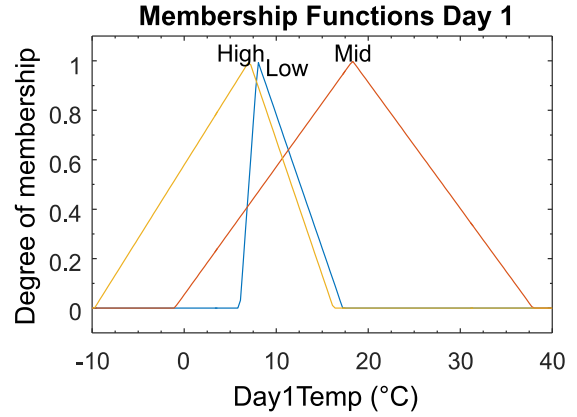

(a)

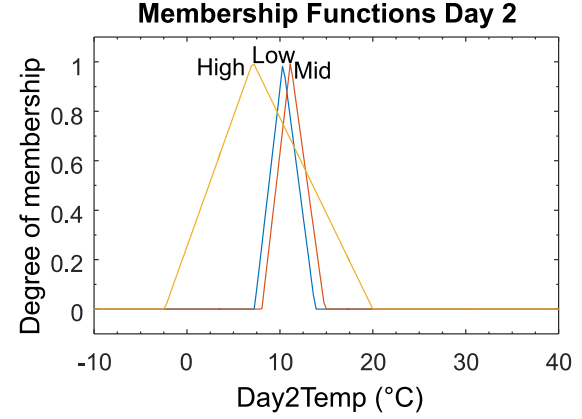

(b)

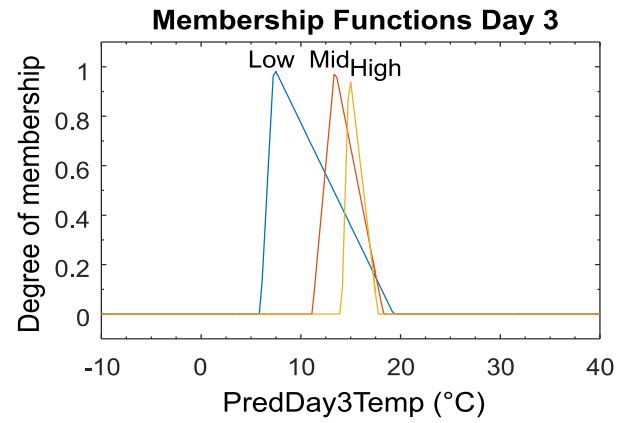

(c)

Figure 11. Membership functions for environment temperature: (a) day 1; (b) day 2; (c) day 3 

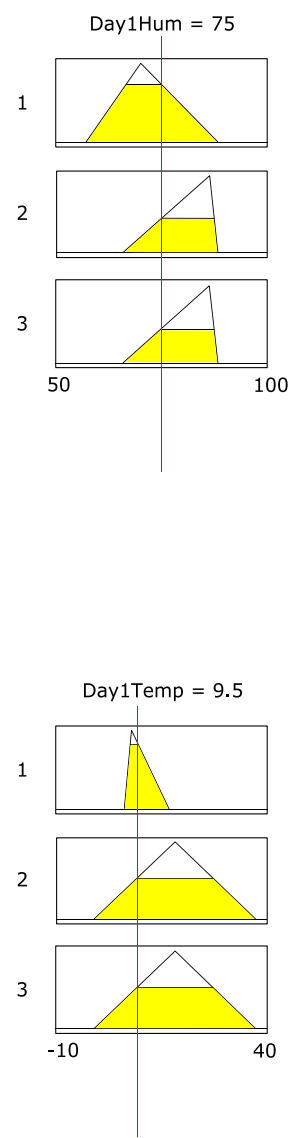
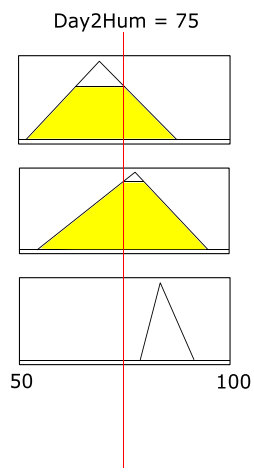

Figure 12. Rules set for environment humidity

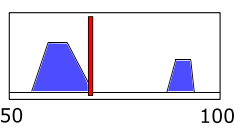

Rules:

If humidity value is low the previous two days, humidity value on the forecast day will be low.

If humidity value oscillates between high and medium the previous two days respectively, humidity value on the forecast day will be medium.

If humidity value is high the previous two days, humidity value on the forecast day will be high.
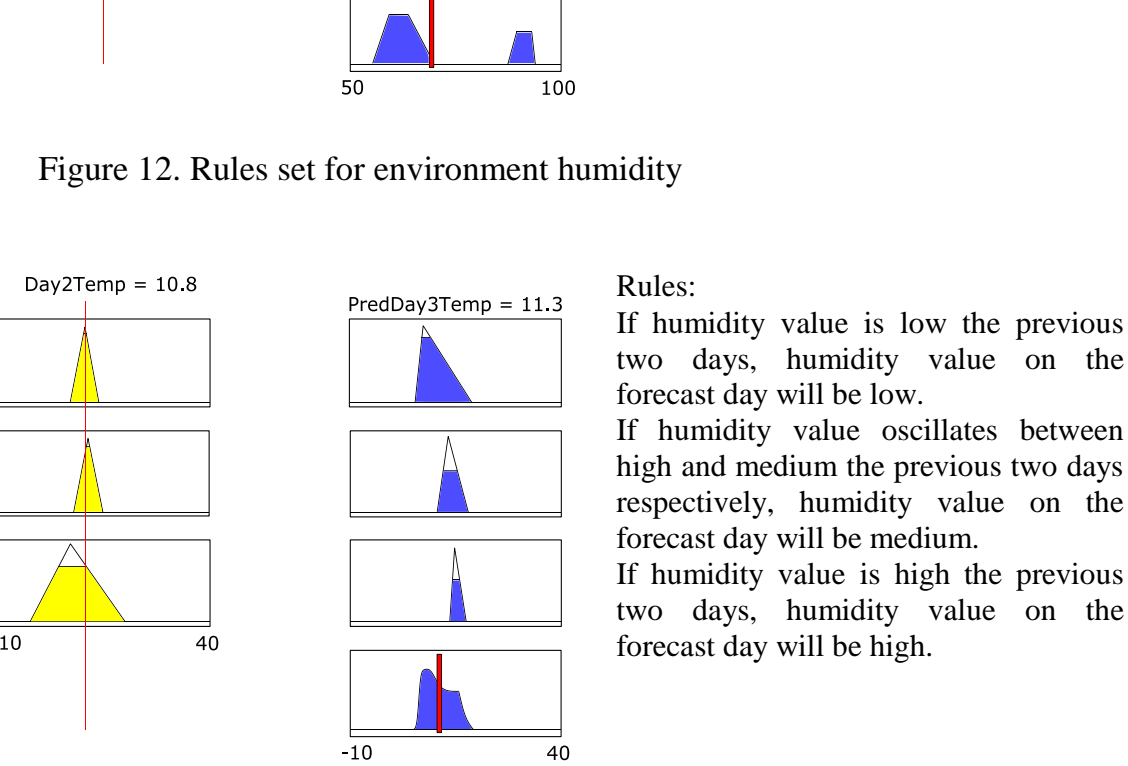

Rules:

If humidity value is low the previous two days, humidity value on the forecast day will be low.

If humidity value oscillates between high and medium the previous two days respectively, humidity value on the forecast day will be medium.

If humidity value is high the previous two days, humidity value on the forecast day will be high.

Figure 13. Rules set for environment temperature

Output Surface: Environment Humidity

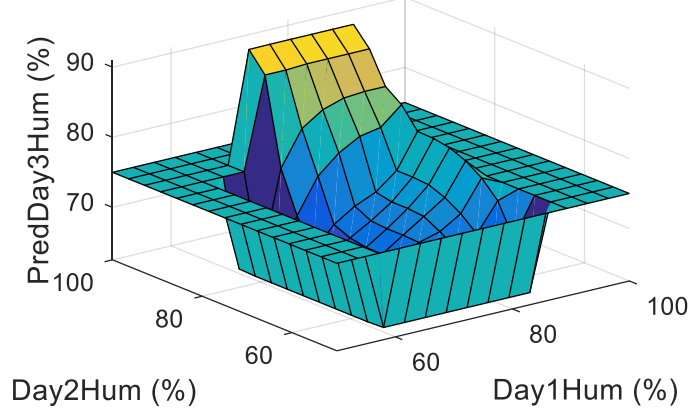

Figure 14. Environment humidity output surface

\section{Output Surface: Environment Temperature}

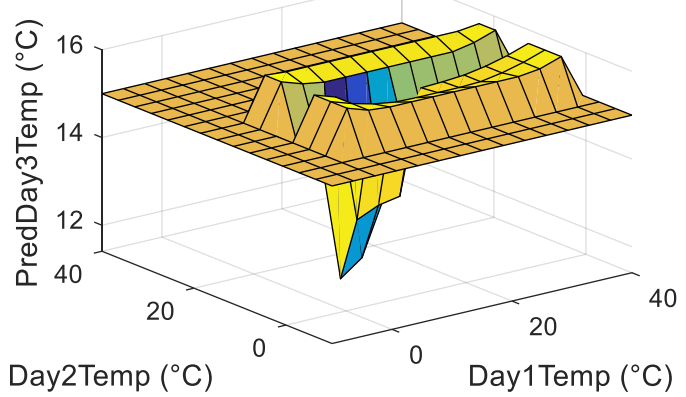

Figure 15. Environment humidity output surface

\section{CONCLUSION}

The use of fuzzy logic to present a solution has great advantages as this system is highly rated for interpretability, furthermore, by relating the obtained values for environment humidity and temperature to qualitative categories as high, medium or low, it allows non-specialized users to understand the system dynamics. Through this research, we have generated two models highly interpretable and accurate to predict the environment humidity and temperature values based on two fuzzy inference systems with three membership functions (low, medium, and high values) each. According to the results, the best prediction performance is obtained when using a Mamdani fuzzy inference system optimized with a Quasi-Newton algorithm: 1.21 E-04 MSE for humidity values and 2.03 E-03 for temperature values. It is important to note that the initial values for this model are attained through an optimization process with a genetic algorithm. 


\section{REFERENCES}

[1] J. Beltrano and D. O. Gimenez, "Cultivo en hidroponía," Editorial de la Universidad Nacional de La Plata (EDULP), 2015

[2] L. L. Sloat et al., "Climate adaptation by crop migration,” Nat. Commun., vol. 11, no. 1, pp. 1-9, 2020.

[3] M. Kang, X. Fan, J. Hua, H. Wang, X. Wang, and F. Wang, "Managing Traditional Solar Greenhouse With CPSS: A Just-for-Fit Philosophy," IEEE Trans. Cybern., vol. 48, no. 12, pp. 3371-3380, 2018.

[4] F. Fourati, "Multiple neural control of a greenhouse," Neurocomputing, vol. 139, pp. 138-144, 2014.

[5] L. E. Nugroho, A. G. H. Pratama, I. W. Mustika, and R. Ferdiana, "Development of monitoring system for smart farming using Progressive Web App," in 2017 9th International Conference on Information Technology and Electrical Engineering (ICITEE), 2017, pp. 1-5.

[6] S. K. Lenka and A. G. Mohapatra, "Gradient Descent with Momentum based Neural Network Pattern Classification for the Prediction of Soil Moisture Content in Precision Agriculture," 2015 IEEE International Symposium on Nanoelectronic and Information Systems, 2015, pp. 63-66.

[7] S. S. Izquierdo and L. R. Izquierdo, "Mamdani Fuzzy Systems for Modelling and Simulation: A Critical Assessment," J. Artif. Soc. Soc. Simul., vol. 21, 2018.

[8] MathWorks Inc., "Mamdani and Sugeno Fuzzy Inference Systems-MATLAB and Simulink," 2019, [Online], Available: https://www.mathworks.com/help/fuzzy/types-of-fuzzy-inference-systems.html

[9] M. Outanoute, A. Lachhab, A. Ed-dahhak, A. Selmani, M. Guerbaoui, and B. Bouchikhi, "A neural network dynamic model for temperature and relative humidity control under greenhouse," 2015 Third International Workshop on RFID And Adaptive Wireless Sensor Networks (RAWSN), 2015, pp. 6-11.

[10] O. C. Da Rocha Neto, A. D. S. Teixeira, A. P. D. S. Braga, C. C. Dos Santos, and R. A. D. O. Leão, “Application of artificial neural networks as an alternative to volumetric water balance in drip irrigation management in watermelon crop," J. Brazilian Assoc. Agric., vol. 35, no. 2, pp. 266-279, 2015.

[11] S. Manabe, J. Smagorinsky, R. F. Strickler, S. Manabe, J. Smagorinsky, and R. F. Strickler, "Simulated Climatology Of A General Circulation Model With A Hydrologic Cycle," Monthly Weather Review, vol. 95, no. 4, pp. 155-169, 1965.

[12] D. B. Jensen, M. S. Torn, and J. Harte, "In our own hands: A strategy for conserving California's biological diversity," University of California Press, 1993.

[13] J. Ballabrera, "Módulo I: Motores de la Biosfera Capítulo 11 Modelos generales de circulación oceánica," 2010.

[14] D. A. Stainforth et al., "Uncertainty in predictions of the climate response to rising levels of greenhouse gases," Nature, vol. 433, no. 7024, pp. 403-406, Jan. 2005.

[15] S. Saha et al., "The NCEP Climate Forecast System Reanalysis," Bull. Am. Meteorol. Soc., vol. 91, no. 8, pp. 1015-1058, 2010.

[16] Goverment of Canada, "Climate models," Canada.ca, 2019. [Online], Available: https://www.canada.ca/en/environment-climate-change/services/climate-change/science-research-data/modelingprojections-analysis/centre-modelling-analysis/models.html

[17] NASA, "Newsroom," NASA Earth Observatory, 2019. [Online], Available: https://earthobservatory.nasa.gov/newsroom,

[18] L. Chen, B. Zhang, F. Yao, and L. Cui, "Modeling and simulation of a solar greenhouse with natural ventilation based on error optimization using fuzzy controller," 2016 35th Chinese Control Conference (CCC), 2016, pp. 2097-2102.

[19] R. S. Pressman, "Software Engineering: A Practitioners Approach,” Novena, McGraw-Hill Education, 2019.

[20] IDEAM, "Pronósticos y Alertas," Instituto de Hidrología, Meteorología y Estudios Ambientales, 2019.

[21] S. Rao, "Engineering Optimization Theory and Practice," Hoboken, NJ: John Wiley and Sons Inc, 2009.

[22] K. Bryan, "Rose-Hulman Institute of Technology," 2017, [Online], Available: https://www.rosehulman.edu/academics/faculty/bryan-kurt-bryan.html

[23] J. Lambers, "MAT 461/561 Numerical Analysis II," 2011, [Online], Available: https://www.math.usm.edu/lambers/mat461/.

[24] MATLAB, "MATLAB - El lenguaje del cálculo técnico-MATLAB \& Simulink," 2019. [Online], Available: https://la.mathworks.com/products/matlab.html.

[25] I. H. Witten, E. Frank, M. Hall, and C. J. Pal, "Data Mining Practical Machine Learning Tools and Techniques," Cambridge, MA: Elsevier Inc, 2017. 